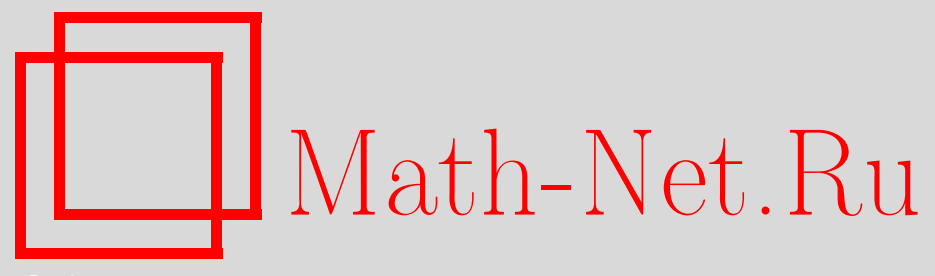

В. П. Радченко, С. В. Горбунов, Метод решения краевой упругопластической задачи о растяжении полосы с концентраторами напряжений с учетом локальных областей пластического разупрочнения материала, Вестн. Сам. гос. техн. ун-та. Сер. Физ.-мат. науки, 2014, выпуск 4(), 98-110

DOI: https://doi.org/10.14498/vsgtu1366

Использование Общероссийского математического портала MathNet.Ru подразумевает, что вы прочитали и согласны с пользовательским соглашением

http: //www.mathnet.ru/rus/agreement

Параметры загрузки:

IP: 3.89 .197 .203

26 апреля 2023 г., 13:39:59

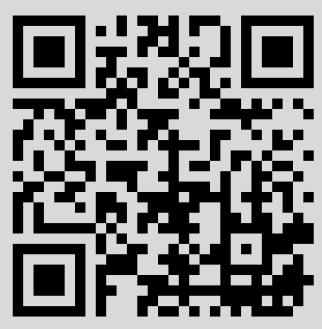




\title{
МЕТОД РЕШЕНИЯ КРАЕВОЙ УПРУГОПЛАСТИЧЕСКОЙ ЗАДАЧИ О РАСТЯЖЕНИИ ПОЛОСЫ С КОНЦЕНТРАТОРАМИ НАПРЯЖЕНИЙ С УЧЕТОМ ЛОКАЛЬНЫХ ОБЛАСТЕЙ ПЛАСТИЧЕСКОГО РАЗУПРОЧНЕНИЯ МАТЕРИАЛА
}

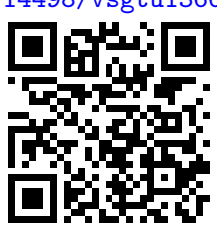

\section{В. П. Радченко, С. В. Горбунов}

Самарский государственный технический университет, Россия, 443100, Самара, ул. Молодогвардейская, 244.

\begin{abstract}
Аннотация
Предложен метод решения связанной краевой задачи деформирования твёрдого тела из пластически разупрочняющегося материала с учётом его повреждённости. Исходными данными для расчёта являются поля деформаций и напряжений, полученные по результатам моделирования поведения условно неповреждённой конструкции при действии фиктивных сил. В предположении эквивалентности деформаций условно неповреждённого и реального тел по соотношениям эндохронной теории пластичности для каждой точки конструкции рассчитывается параметр повреждённости, который связывает компоненты тензора истинных напряжений условно неповреждённой среды и компоненты тензора номинальных напряжений реальной среды. Зная последний тензор, можно вычислить обобщённые силы реальной конструкции и расчётным путём установить области закритического деформирования материала. Решались задачи о растяжении пластин малой толщины, ослабленных центральным круговым отверстием и полукруговыми вырезами. Поля деформаций и истинных напряжений были получены численным расчётом в конечно-элементном пакете и использованы для определения номинальных напряжений реальной конструкции. Построены зоны пластического разупрочнения материала; установлено, что до момента, предшествующего разрушению, в области концентрации напряжений реализуется закритическая стадия, хотя диаграмма «обобщённое перемещение - обобщённая нагрузка» для пластин соответствует стадии пластического упрочнения. Выполнена проверка адекватности метода решения краевой задачи для растягиваемых образцов из сплава АД1 экспериментальным данным. Наблюдается соответствие расчётных и опытных результатов.
\end{abstract}

Ключевые слова: краевая задача, концентратор напряжений, закритическая стадия деформирования, плоское напряжённое состояние, условно

(C) 2014 Самарский государственный технический университет.

Образец для цитирования

Р ад ченко В. П., Горбунов С. В. Метод решения краевой упругопластической задачи о растяжении полосы с концентраторами напряжений с учетом локальных областей пластического разупрочнения материала // Вестн. Сам. гос. техн. ун-та. Сер. Физ.-мат. науки, 2014. № 4 (37). C. 98-110. doi: 10.14498/vsgtu1366.

\section{Сведения об авторах}

Владимир Павлович Радченко (д.ф.-м.н., проф.; radch@samgtu.ru; автор, ведущий переписку), заведующий кафедрой, каф. прикладной математики и информатики.

Сергей Владимирович Горбунов (Ogorbunov0@gmail.com), ассистент, каф. прикладной математики и информатики. 
неповреждённая среда, пластичность, экспериментальная проверка. doi: http://dx.doi.org/10.14498/vsgtu1366

Введение. Повышение несущей способности деталей и элементов конструкций при минимизации их материалоёмкости достигается, в том числе, более полным использованием ресурса материалов, который зачастую определяется их разупрочнением и процессами, связанными с накоплением повреждённости. Поэтому классические способы оценки прочности элементов конструкций из упругопластических материалов, ориентированные на определение напряжённо-деформированного состояния в наиболее нагруженных участках с последующей критериальной оценкой состояния материалов в этих областях, не могут учесть все внутренние ресурсы конструкции с зонами пластического разупрочнения. С точки зрения живучести элементов конструкций важным явлением механического поведения среды является закритическая стадия деформирования (стадия разупрочнения). Поэтому вопросы экспериментального [1-9 и др.] и теоретического [10-17 и др.] исследований закономерностей закритического неупругого деформирования в настоящее время привлекают повышенное внимание с целью более точного прогнозирования процессов разрушения и использования деформационных резервов материалов. Однако методы решения краевых задач для конструкций из пластически разупрочняющихся материалов предложены лишь в простейших случаях (растяжение, кручение, изгиб) [10-12, 15-17], поэтому, по всей видимости, отсутствует и соответствующее программное обеспечение даже в широко известных специализированных вычислительных комплексах для прочностных расчётов.

В настоящей работе излагается способ решения краевых задач для растягиваемых пластин из пластически разупрочняющегося материала с концентраторами напряжений.

\section{1. Метод решения краевой задачи пластического разупрочнения на осно-} ве концепции эквивалентной неповреждённой среды. Предлагается вариант решения связанной задачи неупругого деформирования твёрдого тела из пластически разупрочняющегося (вследствие накопления повреждённости) материала; суть данного метода состоит в следующем. На макроуровне разупрочняющейся реальной среде, описываемой при помощи тензора номинальных напряжений $\sigma_{i j}^{0}$, ставится в соответствие псевдонеповреждённая среда, где вместо классических переменных $\sigma_{i j}^{0}$ используются эффективные (истинные) напряжения $\sigma_{i j}$. Определение эффективных переменных состояния основано на принципе эквивалентных деформаций [18-20]. В соответствии с [17] приведённое (истинное) напряжение $\sigma_{i j}$ связанно с обычным (номинальным напряжением) $\sigma_{i j}^{0}$ зависимостью:

$$
\begin{gathered}
\sigma_{i j}=\sigma_{i j}^{0}(1+\omega), \\
\dot{\omega}=\gamma\left(E^{p}\right)^{m} \sigma_{i j} \dot{e}_{i j}^{p},
\end{gathered}
$$

где $\omega$ - параметр повреждённости; $E^{p}$ - интенсивность пластических деформаций; $\gamma, m$ - параметры степенной аппроксимации; $e_{i j}^{p}$-компоненты тензора пластических деформаций; по повторяющимся индексам $(i, j=1,2,3)$ 
производится суммирование. Тогда уравнения равновесия для повреждённой среды

$$
\sigma_{i j, j}^{0}+X_{i}=0, \quad i, j=1,2,3,
$$

где $X_{i}$ - объёмные силы, могут быть преобразованы к уравнениям равновесия для псевдонеповреждённой среды

$$
\sigma_{i j, j}-Y_{i}=0, \quad i, j=1,2,3,
$$

где $Y_{i}$ - приведённые объёмные силы, которые имеют вид

$$
Y_{i}=\frac{\sigma_{i j}}{1+\omega} \omega, j+X_{i}(1+\omega) .
$$

Таким образом, (3) и (4) являются уравнениями равновесия для эквивалентной среды без повреждений.

Определение параметров $\gamma$ и $m$ в степенной аппроксимации осуществляется на основе одноосной модели, которая имеет вид (при $\sigma_{\text {пр }}=0, \sigma_{\text {пр }}$ - предел упругости) [17]

$$
\begin{aligned}
& \varepsilon=\frac{\sigma}{E}+e^{p} ; \\
& \dot{e}^{p}(t)=\left\{\begin{array}{cc}
\lambda\left(a(\sigma(t))^{n}-e^{p}(t)\right), & a(\sigma(t))^{n}>e^{p}(t), \\
0, & a(\sigma(t))^{n} \leqslant e^{p}(t) ;
\end{array}\right. \\
& \sigma(t)=\sigma^{0}(1+\omega(t)) ; \\
& \dot{\omega}(t)=\gamma\left(e^{p}(t)\right)^{m} \sigma(t) \dot{e}^{p}(t) ; \\
& e^{p}(0)=0, \omega(0)=0 .
\end{aligned}
$$

Здесь $\varepsilon-$ полная деформация, $e^{p}-$ деформация пластичности, $\sigma^{0}, \sigma-$ номинальное и истинное напряжения (соответственно), $\omega-$ скалярный параметр повреждённости, $E$ - модуль упругости, $a, n, \lambda, \gamma, m$ - константы модели, $t$ - параметр нагружения (внутреннее время).

Отметим, что соотношения (5) в координатах $\sigma^{0} \sim \varepsilon$ описывают полную диаграмму деформирования с учётом ниспадающей ветви, а в координатах $\sigma \sim \varepsilon$ диаграмма деформирования является строго монотонно возрастающей. Поэтому предлагается вести упругопластический расчёт в истинных напряжениях, используя любую теорию пластичности для упрочняющегося материала, а пересчёт напряжённо-деформированного состояния для номинальных напряжений $\sigma_{i j}^{0}$ осуществлять на основе соотношений $(1),(2)$. Другими словами, с помощью диаграммы $\sigma \sim \varepsilon$ задаются свойства материала в конечно-элементом пакете, в котором рассчитываются тензоры деформаций $e_{i j}^{p}$ и напряжений $\sigma_{i j}$ условно неповреждённой конструкции, а тензор напряжений $\sigma_{i j}^{0}$ реальной конструкции вычисляется следующим образом:

$$
\sigma_{i j}^{0}=\frac{\sigma_{i j}}{1+\omega}
$$

параметр повреждённости $\omega$ определяется из соотношения (2). 
2. Численное решение и экспериментальная проверка метода. В качестве примера применения предложенного метода на практике решена задача об одноосном равномерном растяжении плоских прямоугольных пластин, ослабленных концентраторами напряжений: центральным круговым отверстием и полукруговыми вырезами. Соответствующие экспериментальные данные для образцов с концентраторами из технического алюминия АД1, изготовленных по ГОСТ 1497-84, были получены на машине Instron 5988 в лаборатории кафедры «Механика» СамГТУ. Фотографии образцов до и после растяжений представлены на рис. 1. Также были проведены испытания сплошного плоского образца, по их результатам построена экспериментальная кривая одноосного упругопластического деформирования материала (штрихпунктирная линия на рис. 2), на основании которой для модели (5) получены теоретические диаграммы одноосного деформирования в координатах $\sigma \sim \varepsilon$ и $\sigma^{0} \sim \varepsilon$ (рис. 2). Параметры модели для описания упругопластической деформации сплава АД1 при $T=20^{\circ} \mathrm{C}$ приведены ниже:

$$
\begin{array}{lll}
E=1.37 \cdot 10^{4} \mathrm{M \Pi а} ; & \sigma_{\text {пр }}=55 \mathrm{M \Pi а}, & n=6.89 ; \\
a=2.09 \cdot 10^{-15}(\mathrm{M \Pi а})^{-n} ; & \gamma=5.22 \cdot 10^{9}(\mathrm{M \Pi а})^{-1} ; & m=14.33 .
\end{array}
$$

Решение соответствующих краевых задач для пластин с концентраторами осуществлялось в программной системе конечно-элементного анализа Ansys на вычислительном кластере «Наука» СамГТУ. Упругие свойства рассматриваемого материала задавались посредством модуля Юнга $E$ и коэффициента Пуассона $\mu=0.3$, пластическое поведение характеризовалось значениями логарифмических деформаций и истинных напряжений в модели не зависящего от скорости деформирования мультилинейного изотропного упрочнения с условием текучести Мизеса. Для построения сетки был выбран конечный элемент Solid183 для режима плоского напряжённого состояния с учётом толщины. В силу наличия у моделируемых образцов двух осей симметрии при решении задачи рассматривались только отсекаемые ими четвертины пластин. Их геометрия, а также схемы задания кинематических и силовых граничных условий представлены на рис. 3 .

На основе полученных в результате расчёта полей пластических деформаций и истинных напряжений по соотношениям (2), (6) были восстановлены поля номинальных напряжений реальной конструкции. Для этих целей на языке APDL (Ansys Parametric Design Language) была написана подпрограмма, в которой уравнение (2) решалось численно методом Эйлера.

На торце $S$ пластины, к которому приложены растягивающие усилия, по найденным номинальным напряжениям вычислялась обобщённая нагрузка

$$
Q=\iint_{S} \sigma_{y}^{0} \mathrm{~d} S
$$

и строилась зависимость её от величины перемещений $u$ этого торца в направлении оси $y$. Полученные расчётные диаграммы «обобщённое перемещение - осевая сила» проведены на рис. $4(a), 5(a)$ сплошными линиями, а штриховыми там же нанесены результаты эксперимента, точками отмечены моменты нагружения, для которых на рис. 4 (b1-b6), 5 (b1-b6) построены поля номинальных напряжений, а на рис. $4(c 1-c 6), 5(c 1-c 6)$ - зоны закритического деформирования для выделенных на схемах пластин (рис. 3) областей А и В. Вне их не было найдено конечных элементов, для которых 

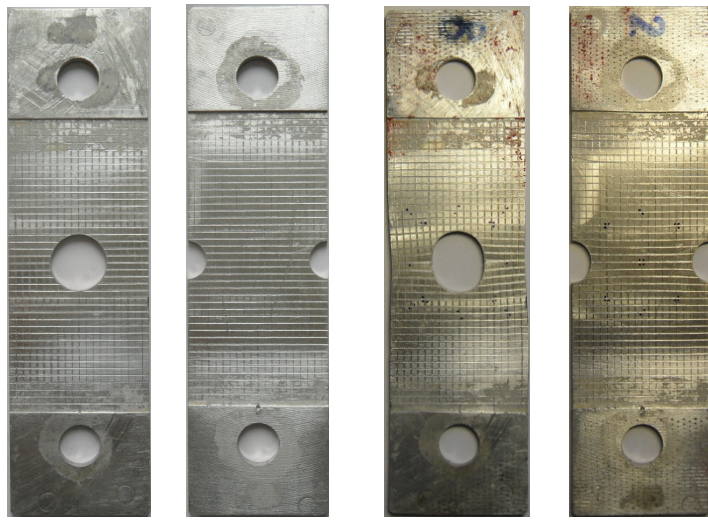

Рис. 1. Фотографии образцов до и после испытаний

[Figure 1. Photographs of the test specimens before and after mechanical testing]

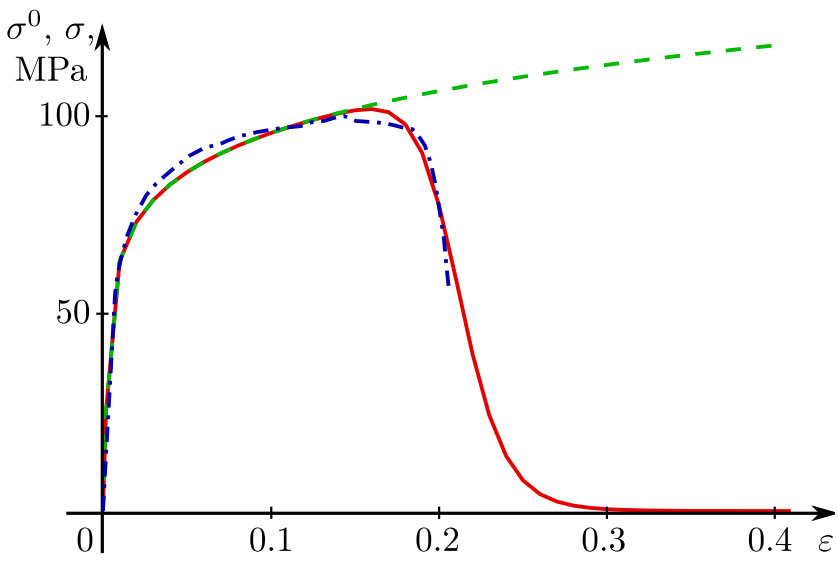

Рис. 2. Кривые упругопластического деформирования сплава АД 1 при $T=20^{\circ} \mathrm{C}$ : расчётные в номинальных (сплошная линия) и истинных (штриховая линия) напряжениях, экспериментальная (штрих-пунктирная линия)

[Figure 2. Elastic-plastic stress-strain curves for AD1 aluminum alloy at $T=20^{\circ} \mathrm{C}$ : calculated in engineering stress (continuous line) and true stress (dashed line), experimental (dot-and-dash line)]
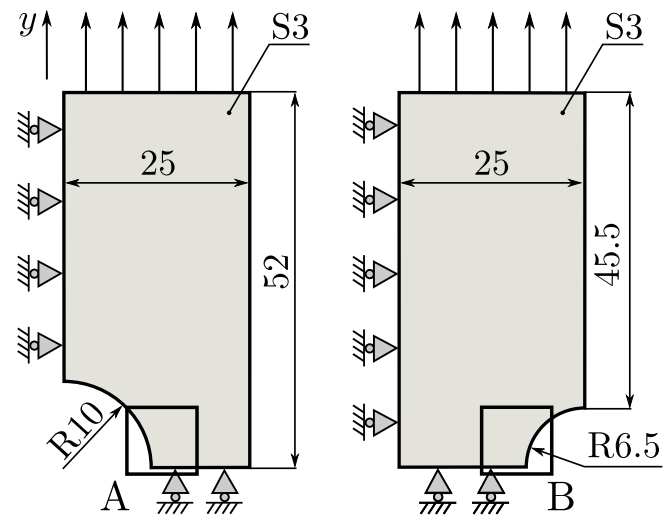

Рис. 3. Схемы образцов

[Figure 3. Sketches of the test specimens] 

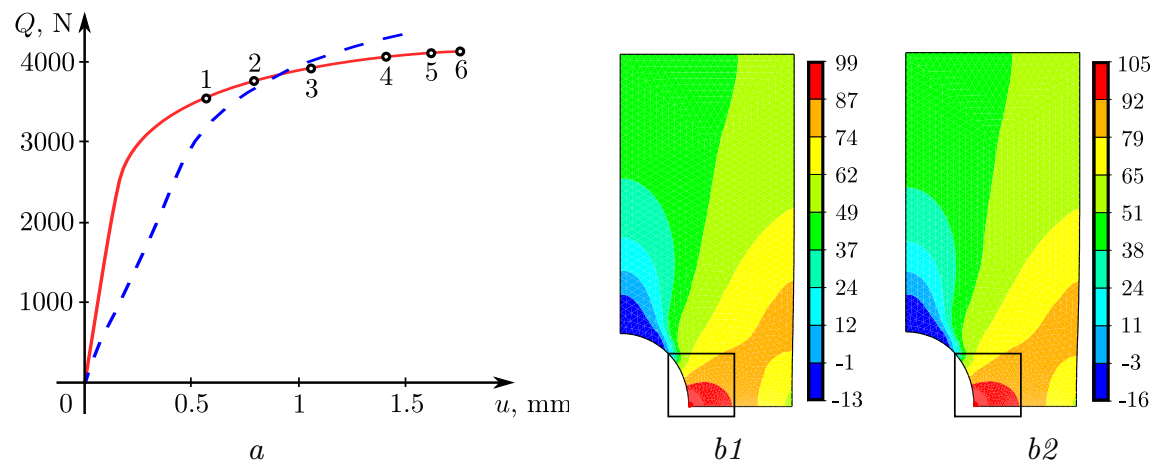

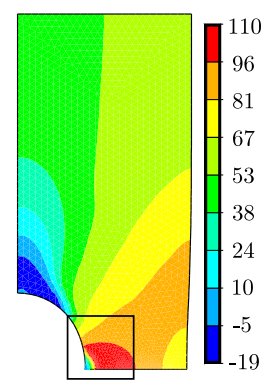

b3

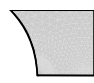

c1

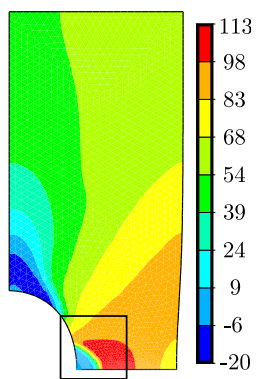

b4

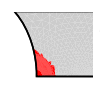

c3 b1

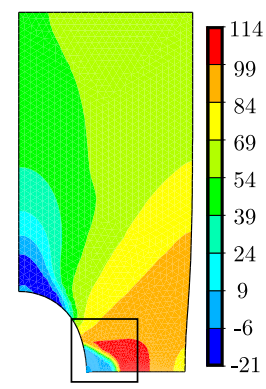

b5

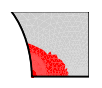

c4 b2

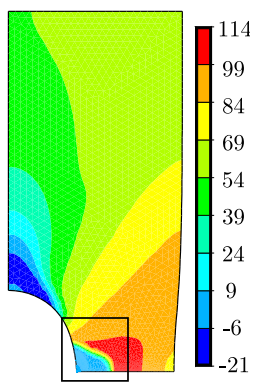

b6

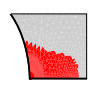

c6

Рис. 4. Расчётная (сплошная линия) и экспериментальная (штриховая линия) диаграммы «обобщённое перемещение - обобщённая нагрузка» для образца с центральным круговым отверстием $(a)$, моментам нагружения 1-6 отвечают поля номинальных напряжений $\sigma_{y}^{0}$ $(b 1-b 6$, соответственно) и области пластического разупрочнения материала $(c 1-c 6$, соответственно)

[Figure 4. Calculated (continuous line) and experimental (dashed line) curves "total displacement - axial force" for the specimen with centric circular hole $(a)$; engineering stress $\sigma_{y}^{0}$ fields $(b 1-b 6)$ and softening plasticity domains $(c 1-c 6)$ correspond to the moments of loading $1-6$ on the calculated curve respectively] 


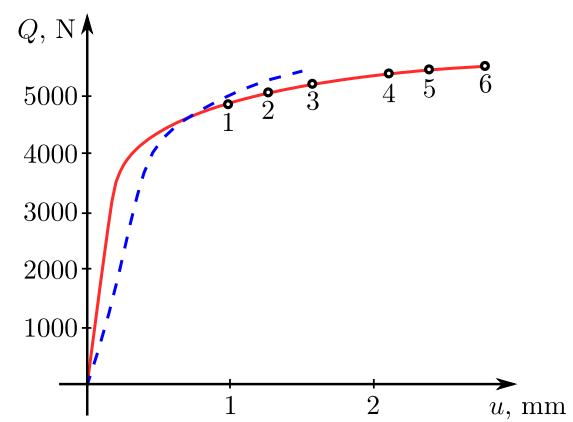

$a$

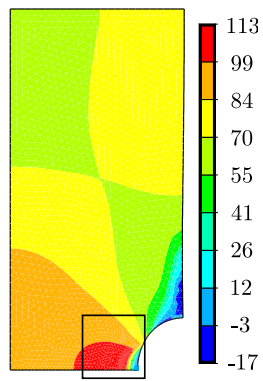

b3

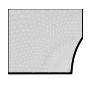

$c 1$

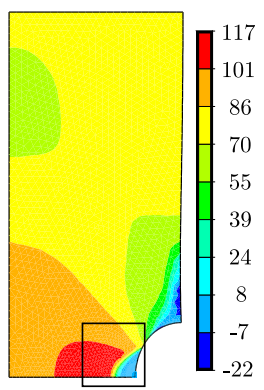

b4

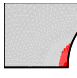

c2

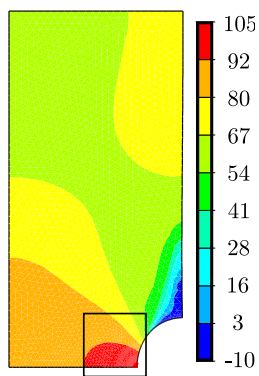

b1

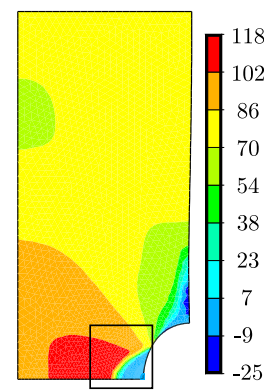

b5

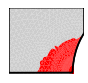

c4

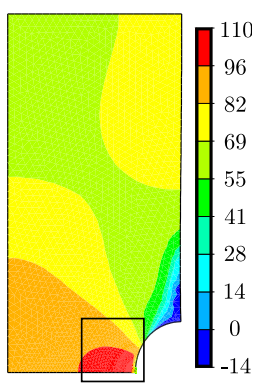

b2

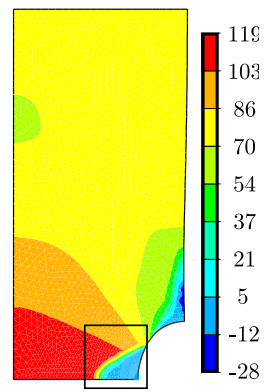

b6

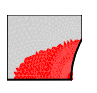

c6

Рис. 5. Расчётная (сплошная линия) и экспериментальная (штриховая линия) диаграммы «обобщённое перемещение - обобщённая нагрузка» для образца с полукруговыми вырезами $(a)$, моментам нагружения 1-6 отвечают поля номинальных напряжений $\sigma_{y}^{0}(b 1-b 6$, соответственно) и области пластического разупрочнения материала ( $c 1-c 6$, соответственно)

[Figure 5. Calculated (continuous line) and experimental (dashed line) curves "total

displacement - axial force" for the specimen with semicircular notches $(a)$; engineering stress $\sigma_{y}^{0}$ fields $(b 1-b 6)$ and softening plasticity domains $(c 1-c 6)$ correspond to the moments of loading $1-6$ on the calculated curve respectively] 


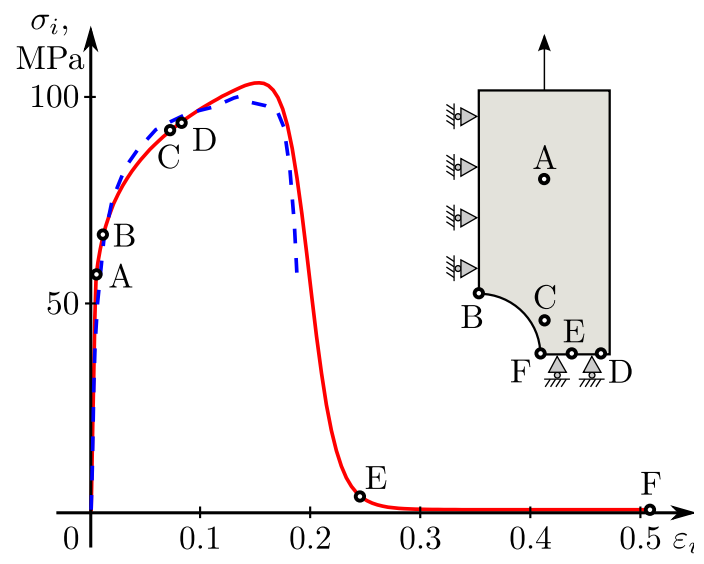

Рис. 6. Расчётные (сплошные линии) кривые зависимости интенсивности тензора номинальных напряжений от интенсивности тензора полных деформаций в точках пластины с центральным круговым отверстием и экспериментальная (штриховая линия) диаграмма одноосного упругопластического деформирования сплава АД1 при $T=20^{\circ} \mathrm{C}$ (пояснения в тексте)

[Figure 6. Calculated (continuous lines) curves of dependency between engineering stress tensor intensity and total strain tensor intensity at points of the plate with centric circular hole and experimental (dashed line) uniaxial elastic-plastic stress-strain curve for $\mathrm{AD} 1$ aluminum alloy at $\left.T=20^{\circ} \mathrm{C}\right]$

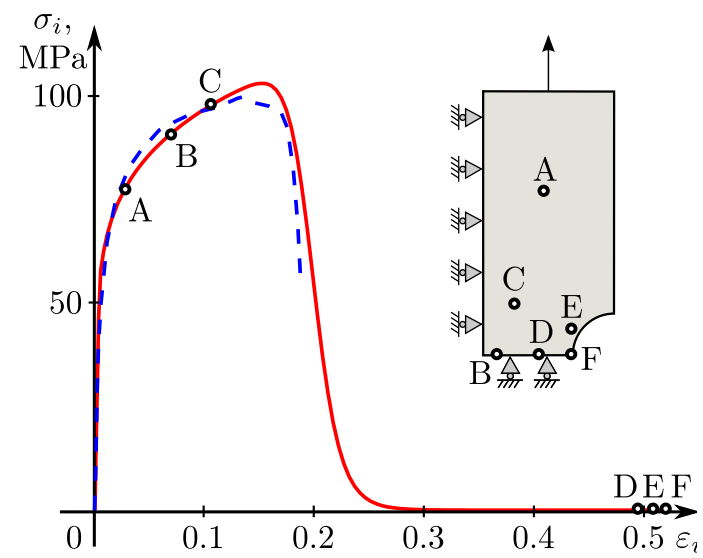

Рис. 7. Расчётные (сплошные линии) кривые зависимости интенсивности тензора номинальных напряжений от интенсивности тензора полных деформаций в точках пластины с полукруговыми вырезами и экспериментальная (штриховая линия) диаграмма одноосного упругопластического деформирования сплава АД 1 при $T=20^{\circ} \mathrm{C}$ (пояснения в тексте)

[Figure 7. Calculated (continuous lines) curves of dependency between engineering stress tensor intensity and total strain tensor intensity at points of the plate with semicircular notches and experimental (dashed line) uniaxial elastic-plastic stress-strain curve for AD1 aluminum alloy at $T=20^{\circ} \mathrm{C}$ ] 
диаграмма зависимости интенсивности тензора номинальных напряжений $\sigma_{i}^{0}$ от интенсивности тензора полных деформаций $\varepsilon_{i}$ имела бы соответствующий разупрочнению материала ниспадающий участок. На рис. 6, 7 упомянутая связь $\sigma_{i}^{0}\left(\varepsilon_{i}\right)$ в нескольких обозначенных там же на схемах пластин точках представлена графически сплошными линиями, штриховыми же для сравнения приведены результаты одноосного эксперимента. Расчётные кривые зависимости интенсивностей напряжений и деформаций в силу однородности материала накладываются друг на друга, и каждая из них обрывается в момент нагружения, соответствующий последней сходящейся итерации численного решения методом конечных элементов (метка 6 на рис. $4(a), 5(a))$. Точки обрыва подписаны так же, как они обозначены на схемах пластин.

Выводы. Получена удовлетворительная корреляция расчётных и опытных данных зависимости «обобщённое перемещение - осевая сила» (рис. 4 ( $a$ ), $5(a))$, а их незначительные расхождения могут быть следствием систематических ошибок как в методике эксперимента, так и в процедуре расчёта. Для выявления причин такого несоответствия необходимо проведение дополнительных испытаний реальных образцов. Несмотря на этот изъян, в работе показана возможность решения краевой задачи упругопластического деформирования в случае сложного напряжённого состояния с определением зон разупрочнения материала. Исходными данными при этом являются лишь одноосные диаграммы деформирования. На примере конкретных задач о растяжении ослабленных отверстием и вырезами пластин установлено, что в момент, предшествующий их разрушению, в области концентрации напряжений реализуется закритическая стадия деформирования (в окрестности вершины концентратора интенсивность номинальных напряжений $\sigma_{i}^{0}$ стремится к нулю при увеличении растягивающей силы), в то время как диаграмма «обобщённое перемещение - осевая нагрузка» соответствует стадии пластического упрочнения материала.

Следует отметить, что аналогичный подход был применён к решению задачи о растяжении стержня кругового сечения с концентраторами типа выточек в [21], но там связь между эффективными и истинными напряжениями использована в форме Работнова-Качанова.

Благодарности. Работа выполнена при поддержке РФФИ (проект № 13-01-00699-а).

\section{ORCID}

Владимир Павлович Радченко: http://orcid.org/0000-0003-4168-9660

Сергей Владимирович Горбунов: http://orcid.org/0000-0003-1986-9243

\section{БИБЛИОГРАФИЧЕСКИЙ СПИСОК}

1. Быля О. И., Васин Р. А. Деформирование сплавов в режиме сверхпластичности и близких к нему режимах// Известия Тульского государственного университета. Eстественные науки, 2011. №2. С. 116-127.

2. Васин Р. А., Еникеев Ф. У., Мазурский М. И. О материалах с падающей диаграммой // Изв. АН СССР. МТТ, 1995. №2. С. 181-182.

3. Лебедев А. А., Чаусов Н. Г., Марусий О. И., Евецкий Ю. Л., Гришай Г. Х., Гриненко Б. Г. Кинетика разрушения листовой аустенитной стали на заключительной стадии деформирования // Проблемы прочности, 1989. №3. С. 16-21.

4. Миронов В. И. Свойства материала в реологически неустойчивом состоянии // Заводская лаборатория. Диагностика материалов, 2002. Т. 68, № 10. С. 47-52. 
5. Стружанов В. В., Миронов В. И. Деформачионное разупрочнение материала в элементах конструкиий. Екатеринбург: УрО РАН, 1995. 191 с.

6. Чаусов Н. Г., Засимчук У. Э., Маркашова Л. И., Вильдеман В. Э., Турчак Т. В., Пилипенко А. П., Парац В. М. Особенности деформирования пластичных материалов при динамических неравновесных процессах // Заводская лаборатория. Диагностика материалов, 2009. №6. С. 52-59.

7. Faleskog J., Barsoum I. Tension-torsion fracture experiments. Part I: Experiments and a procedure to evaluate the equivalent plastic strain // International Journal of Solids and Structures, 2013. vol.50, no. 25-26. pp. 4241-4257. doi: 10.1016/j.ijsolstr.2013. 08.029.

8. Вильдеман В. Э., Третьяков М. П. Испытания материалов с построением полных диаграмм деформирования // Проблемы машиностроения и надёжности машин, 2013. № 2. С. 93-98.

9. Вильдеман В. Э., Ломакин Е. В., Третьяков М. П. Закритическое деформирование сталей при плоском напряжённом состоянии // Изв. РАН. МТT, 2014. № 1. С. 26-36.

10. Жижерин С. В., Стружанов В. В., Миронов В. И. Итерационные методы расчёта напряжений при чистом изгибе балки из повреждающегося материала // Вычисл. технол., 2001. T. 6, № 5. C. 24-33.

11. Стружанов В. В. Определение инкрементальных модулей по результатам испытаний на растяжение с построением полной диаграммы // Вестн. Сам. гос. техн. ун-та. Сер. Физ.-мат. науки, 2008. №1(16). С. 160-163. doi: 10.14498/vsgtu591.

12. Стружанов В. В. Свойства разупрочняющихся материалов и определяющие соотношения при одноосном напряженном состоянии // Вестн. Сам. гос. техн. ун-та. Сер. Физ.-мат. науки, 2007. № 2(15). С. 69-78. doi: 10.14498/vsgtu532.

13. Вильдеман В. Э., Ильиных А. В. Моделирование процессов структурного разрушения и масштабных эффектов разупрочнения на закритической стадии деформирования неоднородных сред // Физ. мезомех., 2007. Т. 10, № 4. С. 23-29.

14. Ильиных А. В. Численное моделирование процессов структурного разрушения зернистых композитов с изотропными элементами структуры // Вестн. Сам. гос. техн. ун-та. Сер. Физ.-мат. науки, 2011. № 2(23). С. 101-106. doi: 10.14498/vsgtu947.

15. Андреева Е. А. Решение одномерных задач пластичности для разупрочняющегося материала // Вестн. Сам. гос. техн. ун-та. Сер. Физ.-мат. науки, 2008. №2(17). С. 152160. doi: 10.14498/vsgtu642.

16. Горбунов С. В. Вариант решения краевой задачи о чистом изгибе балки из пластически разупрочняющегося материала/ Труды девятой Всероссийской научной конференции с международным участием. Ч. 1: Математические модели механики, прочности и надёжности элементов конструкций / Матем. моделирование и краев. задачи. Самара: СамГТУ, 2013. С. 92-96.

17. Радченко В. П., Еремин Ю. А. Реологическое деформирование и разрушение материалов и элементов конструкиий. М.: Машиностроение-1, 2004. 264 с.

18. Lemaitre J. Evolution of dissipation and damage in metals submitted to dynamic loading / Proc. I.C.M.. vol. I (Kyoto, Japan), 1971. pp. 151-157.

19. Lemaitre J., Chaboche J. L. Aspect phénoménologique de la rupture par endommagement // J. de Mécanique Appliqué, 1978. vol. 2, no. 3. pp. 317-365 (In French).

20. Работнов Ю. Н. Ползучесть элементов конструкиий. М.: Наука, 1966. 752 с.

21. Адеянов И. Е., Клебанов Я. М. Влияние эффекта разрушения материала на условия разупрочнения / Труды шестой Всероссийской научной конференции с международным участием (1-4 июня 2009 г.). Часть 1: Математические модели механики, прочности и надёжности элементов конструкций / Матем. моделирование и краев. задачи. Самара: СамГТУ, 2009. С. 10-12.

Поступила в редакцию 13/XI/2014;

в окончательном варианте - 09/XII/2014;

принята в печать - 11/XII/2014. 
Vestn. Samar. Gos. Techn. Un-ta. Ser. Fiz.-mat. nauki

[J. Samara State Tech. Univ., Ser. Phys. \& Math. Sci.] 2014. Issue 4 (37). Pp. 98-110

ISSN: 2310-7081 (online), 1991-8615 (print)

doi: http://dx.doi.org/10.14498/vsgtu1366

MSC: 74C05; 74G70, 74R20

\title{
THE METHOD OF SOLUTION OF THE ELASTIC-PLASTIC BOUNDARY VALUE PROBLEM OF TENSION OF STRIP WITH STRESS RAISERS WITH ALLOWANCE FOR LOCAL DOMAINS OF SOFTENING PLASTICITY OF MATERIAL
}

\author{
V. P. Radchenko, S. V. Gorbunov
}

Samara State Technical University,

244, Molodogvardeyskaya st., Samara, 443100, Russian Federation.

\begin{abstract}
The way of solution of the coupled boundary value problem of solid body deformation for the case of a plastically softening material is offered. The strain and stress fields obtained by the simulated undamaged construction behavior modeling under the action of fictitious forces are used as basic data for calculation. The equivalence of simulated undamaged medium strains and real medium strains is supposed. At each point of construction the damage parameter $\omega$ is calculated by means of constitutive relations of the endochronic plasticity theory. This damage parameter associates the components of the true stress tensor $\sigma_{i j}$ of simulated undamaged medium and the engineering stress tensor $\sigma_{i j}^{0}$ of real medium by $\sigma_{i j}^{0}=\sigma_{i j} /(1+\omega)$. Using the tensor $\sigma_{i j}^{0}$ we can calculate the generalized forces of real construction. The problems of tension of the plates weakened with centric circular hole and semicircular notches are solved and the necessary experiments are conducted. The strain and true stress fields are obtained by numerical calculation at the finite element analysis software and are used for the engineering stress of real construction computation according to the foregoing expression. Softening plasticity domains are plotted. It is found that at the moment before failure the stage of post critical deformation is implementing in the region of stress concentration, although the curve "total displacement - axial force" corresponds to the stage of plastic hardening.
\end{abstract}

Keywords: boundary value problem, stress raiser, post critical deformation, plane stress state, simulated undamaged medium, plasticity, experimental proof.

doi: http://dx.doi.org/10.14498/vsgtu1366

(C) 2014 Samara State Technical University.

\section{How to cite Reference}

Radchenko V. P., Gorbunov S. V. The method of solution of the elastic-plastic boundary value problem of tension of strip with stress raisers with allowance for local domains of softening plasticity of material, Vestn. Samar. Gos. Tekhn. Univ., Ser. Fiz.-Mat. Nauki [J. Samara State Tech. Univ., Ser. Phys. \& Math. Sci.], 2014, no. 4(37), pp. 98-110. doi: 10.14498/vsgtu1366. (In Russian)

\section{Authors Details}

Vladimir P. Radchenko (Dr. Phys. \& Math. Sci., Professor; radch@samgtu.ru; Corresponding Author), Head of Department, Dept. of Applied Mathematics \& Computer Science.

Sergey V. Gorbunov (Ogorbunov0@gmail.com), Assistant, Dept. of Applied Mathematics \& Computer Science. 
Acknowledgments. This work was supported by Russian Foundation for Basic Research (Project No. 13-01-00699-a).

\section{ORCID \\ Vladimir P. Radchenko: http://orcid.org/0000-0003-4168-9660 \\ Sergey V. Gorbunov: http://orcid.org/0000-0003-1986-9243}

\section{REFERENCES}

1. Bylia O. I., Vasin R. A. Behavior of alloys in superplastic and near to superplastics regimes of deforming, Izvestiia Tul'skogo gosudarstvennogo universiteta. Estestvennye nauki, 2011, no. 2, pp. 116-127 (In Russian).

2. Vasin R. A., Enikeev F. U., Mazurskii M. I. Materials with a Falling Diagram, Mech. Solids, 1995, no. 2, pp. 168-169.

3. Lebedev A. A., Chausov N. G., Marusii O. I., Evetskii Yu. L., Grishai G. Kh., Grinenko B. G. Failure kinetics for sheet austenitic steels in the final stage of deformation, Strength of Materials, 1989, vol. 21, no.3, pp. 285-290. doi: 10.1007/bf01529176.

4. Mironov V. I. Properties of the material in rheologically unstable state, Zavodskaia Laboratoriia. Diagnostika Materialov, 2002, vol.68, no. 10, pp. 47-52 (In Russian).

5. Struzhanov V. V., Mironov V. I. Deformational Softening of Material in Structural Elements. Ekaterinburg, Ural Branch of Russian Academy of Sciences, 1995, 191 pp. (In Russian)

6. Chausov N. G., Zasimchuk U. E., Markashova L. I., Vil'deman V. E., Turchak T. V., Pilipenko A. P., Parats V. M. Features of plastic deformation of materials under dynamic non-equilibrium processes, Zavodskaia Laboratoriia. Diagnostika Materialov, 2009, no. 6, pp. 52-59 (In Russian).

7. Faleskog J., Barsoum I. Tension-torsion fracture experiments. Part I: Experiments and a procedure to evaluate the equivalent plastic strain, International Journal of Solids and Structures, 2013, vol.50, no. 25-26, pp. 4241-4257. doi: 10.1016/j.ijsolstr.2013. 08.029.

8. Vil'demann V. E., Tretyakov M. P. Material testing by plotting total deformation curves, J. Mach. Manuf. Reliab., 2013, vol.42, no. 2, pp. 166-170. doi: 10.3103/ S1052618813010159.

9. Wildemann V. E., Lomakin E. V., Tretyakov M. P. Postcritical deformation of steels in plane stress state, Mech. Solids, 2014, vol. 49, no. 1, pp. 18-26. doi : 10.3103/S0025654414010038.

10. Zhizherin S. V., Struzhanov V. V., Mironov V. I. Iterative methods of stress calculation in pure bending of beams of damageable material, Vychisl. Tekhnol., 2001, vol.6, no. 5, pp. 24-33 (In Russian).

11. Struzhanov V. V. Determination of incremental modules on base of tension test results with full diagram construction, Vestn. Samar. Gos. Tekhn. Univ. Ser. Fiz.-Mat. Nauki, 2008, no. 1(16), pp. 160-163 (In Russian). doi: 10.14498/vsgtu591.

12. Struzhanov V. V. The properties of softening materials and constitutive relations under uniaxial stress state, Vestn. Samar. Gos. Tekhn. Univ. Ser. Fiz.-Mat. Nauki, 2007, no. 2(15), pp. 69-78 (In Russian). doi : 10.14498/vsgtu532.

13. Vil'deman V. E., Il'inykh A. V. Simulation of structural failure and scale effects of softening at the post-critical deformation stage in heterogeneous media, Fiz. Mezomekh., 2007, vol. 10, no. 4, pp. 23-29 (In Russian).

14. Ilinykh A. V. Numerical modeling of structural fracture processes for granular composites with isotropic elements of structure, Vestn. Samar. Gos. Tekhn. Univ. Ser. Fiz.-Mat. Nauki, 2011, no. 2(23), pp. 101-106 (In Russian). doi: 10.14498/vsgtu947.

15. Andreeva E. A. Solution of one-dimensional softening materials plasticity problems, Vestn. Samar. Gos. Tekhn. Univ. Ser. Fiz.-Mat. Nauki, 2008, no. 2(17), pp. 152-160 (In Russian). doi: 10.14498/vsgtu642.

16. Gorbunov S. V. The way of solution of the boundary value problem of a pure bending of a beam from plastically softening material, Proceedings of the Ninth All-Russian Scientific 
Conference with international participation. Part 1, Matem. Mod. Kraev. Zadachi. Samara, Samara State Technical Univ., 2013, pp. 92-96 (In Russian).

17. Radchenko V. P., Eremin Iu. A. Reologicheskoe deformirovanie i razrushenie materialov $i$ elementov konstruktsii [Rheological Deformation and Failure of Materials and Structural Elements]. Moscow, Mashinostroenie-1, 2004, 264 pp. (In Russian)

18. Lemaitre J. Evolution of dissipation and damage in metals submitted to dynamic loading, Proc. I.C.M. (Kyoto, Japan), I, 1971, pp. 151-157.

19. Lemaitre J., Chaboche J. L. Aspect phénoménologique de la rupture par endommagement, J. de Mécanique Appliqué, 1978, vol. 2, no. 3, pp. 317-365 (In French).

20. Rabotnov Yu. N. Polzuchest' elementov konstruktsii [Creep of Structural Elements]. Moscow, Nauka, 1966, 752 pp. (In Russian)

21. Adeyanov I. E., Klebanov Ya. M. Influence of the material damage effect on the softening factor, Proceedings of the Sixth All-Russian Scientific Conference with international participation (1-4 June 2009). Part 1, Matem. Mod. Kraev. Zadachi. Samara, Samara State Technical Univ., 2009, pp. 10-12 (In Russian).

Received 13/XI/2014;

received in revised form $09 / \mathrm{XII} / 2014$;

accepted $11 / \mathrm{XII} / 2014$. 